\title{
Node Density-Based Adaptive Routing Scheme for Disruption Tolerant Networks
}

\author{
M. Chuah, P. Yang \\ Department of Computer Science \& Engineering \\ Department of Computer Science \& Engineering \\ Lehigh University \\ chuah@cse.lehigh.edu, pey204@lehigh.edu
}

\begin{abstract}
Traditional ad hoc routing protocols do not work in intermittently connected networks since end-to-end paths may not exist in such networks. Hence, routing mechanisms that can withstand disruptions need to be designed. A store-and-forward approach has been proposed for disruption tolerant networks. Recently, several approaches have been proposed for unicast routing in disruption-prone networks e.g. the 2-hop relay approach, delivery probability based routing, and message ferrying. In our earlier paper, we have evaluated a combined multihop and message ferrying approach in disruption tolerant networks. In that paper, we assume that a special node is designated to be a message ferry. A more flexible approach is to let regular nodes volunteer to be message ferries when network dynamics mandate the presence of such ferries to ensure communications. Thus, in this paper, we design a nodedensity based adaptive routing (NDBAR) scheme that allows regular nodes to volunteer to be message ferries when there are very few nodes around them to ensure the feasibility of continued communications. Our simulation results indicate that our NDBAR scheme can achieve the highest delivery ratio in very sparse networks that are prone to frequent disruptions.
\end{abstract}

Keywords-disruption tolerant networking; adaptive routing; node-density; 2 hop relay

\section{INTRODUCTION}

Packet-switched network communication has been studied for decades. Important progress has been made in robustness and scalability in the TCP/IP protocol suite based primarily on principles of end-to-end protocols and services [9]. However, there are many scenarios in which an end-to-end connection is not guaranteed or even possible, and so an intermediary is needed, perhaps to translate between protocols or to provide temporary storage (e.g., in mail servers). In these cases, without such intermediaries, communication would fail. In other cases, communication may fail not because of a lack of instantaneous connection, but because the connection properties fall beyond the expected bounds (excessive round-trip-time or high packet loss probability).

Solutions have been proposed to deal with some specific situations, e.g., using link layer retransmissions to deal with high packet loss probability in wireless environments [4]. However, these solutions still do not work in situations where there are no end-to-end paths. DakNet [3] deploys physical transport devices, e.g., buses and motorcycles, to carry mobile access points between village kiosks and hubs with Internet connectivity so that the data carried by the physical transport devices can be automatically uploaded and/or downloaded when the physical transport devices are in the wireless communication range of a kiosk or a hub. Similar techniques are proposed in [1],[2]. In the past year, considerable amount of research focusing on delay/disruption-tolerant networking and communications has been published (e.g.[5],[6], [13],[15]). DieselNet [14] is a vehicular-based disruption tolerant network where connections between nodes are short-lived and occasional. A common approach used to address delays and disruptions is via the use of a storeand-forward mechanism similar to electronic mail [10]. This makes communication possible, even when an instantaneous end-to-end path does not exist.

Several routing schemes have been proposed for DTNs. They can be categorized into three categories: (i) using message ferries or data mules to connect partitioned nodes [15],[20], (ii) using history-based information to estimate delivery probability of peers and pass the message to the peer that can best deliver the message [22], [26], and (iii) using 2-hop relay forwarding schemes where a source can send multiple copies to different relay nodes and have the relay nodes deliver to the destination when they encounter the destination [19],[21].

In our earlier work [25], we have evaluated the performance of a multihop routing scheme with custody transfer feature in a single domain DTN. We also have explored using message ferrying and high-power backhaul links for interdomain message delivery. Our work revealed that in a single domain environment, even with the custody transfer feature, the delivery ratio drops when the nodes are sparsely connected. So, in this paper, we propose a node-density based adaptive routing (NDBAR) scheme that provides better performance than previous approaches. 
This paper is organized as follows: In Section II, we summarized related work. In Section III, we describe our node-density based adaptive routing scheme. In Section IV, we describe our simulation models. We also present and discuss our simulation results. We conclude in Section V.

\section{RELATED WORK}

Three categories of forwarding schemes have been proposed for DTNs. In the first category [20], the authors propose to use message ferries or data mules to gather data from stationary sources and deliver them to their destinations. However, nodes that move can be message carriers themselves without having to resort to special message ferries. In the second category [22],[26], the authors propose using history-based routing where each node maintains a utility value for every other node in the network, based on a timer indicating the time elapsed since the two nodes last encountered each other. These utility values which carry indirect information about relative node locations, get diffused through nodes' mobility. Nodes forward message copies only to those nodes with a higher utility for the message's destination. For example in [22], the authors propose a probabilistic metric called delivery predictability at every node for each known destination. This metric indicates how likely it is that a node will be able to deliver a message to each destination. The delivery predictability ages with time and also has a transitive property i.e. a node $\mathrm{A}$ that encounters node $\mathrm{B}$ which encounters node $\mathrm{C}$ allows node $\mathrm{A}$ to update its delivery predictability to node C based on its (A's) delivery predictability to node $\mathrm{B}$ and node B's delivery predictability to node C. In [22], a node will forward a message to another node it encounters if that node has a higher delivery predictability to the destination than itself. Such a scheme was shown to produce better performance than epidemic routing [24].

In the third category [19], [23], the authors propose using a 2-hop relay forwarding scheme where the source sends multiple copies (e.g. different erasure coding blocks) to different relaying nodes and the relaying nodes will deliver the copies they have to the destination node when they encounter the destination node. Again, such strategy will achieve small transmission overhead but may not enjoy high delivery ratio for messages with short deadlines. In this paper, we simulate scenarios similar to those reported in [22], [19] so that we can compare the transmission overhead and the delivery ratios of these different schemes with the schemes we design.

\section{NDBAR SCHEME}

In [25], we have evaluated the performance of multihop routing protocol in a DTN scenario where 40 nodes were distributed over a geographical area of $1000 \times 1000$ to $4000 \times 4000 \mathrm{~m}^{2}$ (assuming a transmission range of $250 \mathrm{~m}^{2}$ ). A DSR-like multihop routing protocol [7] is enhanced with custody transfer feature [18]. Our simulation results indicate that when the node density drops below $4.4 \times 10^{-6}$ (equivalent to finding only one neighbor within the transmission range), the delivery drops significantly despite the custody transfer feature. Table 1 shows the simulation results for the scenarios with 40 nodes distributed over $3000 \times 3000 \mathrm{~m}^{2}$ and $4000 \times 4000 \mathrm{~m}^{2}$. We see that the achievable delivery ratio is only $54.3 \%$ and $18.3 \%$ respectively even with the custody transfer feature turned on. Thus, to improve on the delivery ratio, we design the node-density based adaptive routing (NDBAR) scheme where nodes can turn into message ferries when they detect that the node density around them drops below a certain threshold.

Table 1: Performance of the multihop approach with custody transfer

\begin{tabular}{|c|c|c|c|c|c|}
\hline $\begin{array}{c}\text { Simulation } \\
\text { Area }\end{array}$ & $\begin{array}{c}\text { Delivery } \\
\text { Ratio }\end{array}$ & $\begin{array}{c}\text { Avg } \\
\text { Dly }\end{array}$ & $\begin{array}{c}95 \% \\
\text { Pkt } \\
\text { Dly }\end{array}$ & Overhead & $\begin{array}{c}\text { Hop } \\
\text { count }\end{array}$ \\
\hline $3000 \times 3000$ & $54.6 \%$ & 1829 & 3500 & 2.1 & 1.1 \\
\hline $4000 \times 4000$ & $18.3 \%$ & 932 & 1500 & 0.8 & 1 \\
\hline
\end{tabular}

In NDBAR scheme, we assume that each node periodically (e.g. every 20 seconds) broadcasts a neighbor discovery message to estimate $n_{d}$, the number of neighbors it has. When $\mathrm{n}_{\mathrm{d}}$ drops below a certain threshold $\mathrm{K}$, then that node will set a flag so that it will relay any future route-request message that it receives using high-power transmission. Any node that receives a high-power route request will take note of this fact, and will issue a high-power route reply when it hears from downstream nodes later. The high-power route reply message contains information about the location and speed of the node that replies. The previous-hop node that receives this reply will keep a record of this information so that if this route is chosen for packet delivery, the previous hop node will travel towards the next-hop node so that the data relay can be conducted using regular power transmission.

In Figure 1 below, we illustrate how NDBAR scheme works via an example. The source node, S, broadcasts a route request at regular power. Nodes $\mathrm{n}_{7}, \mathrm{n}_{4}$, and $\mathrm{n}_{8}$ hear this route request and will re-broadcast the 
route request using regular power. Node $\mathrm{n}_{4}$ 's rebroadcast is heard by nodes $n_{9}, n_{3}$, and $n_{8}$. Similarly, node $n_{9}$ 's rebroadcast is heard by nodes $\mathrm{n}_{11}$ and $\mathrm{n}_{1}$. Node $\mathrm{n}_{10}$ realizes that its observed number of neighbors is 1 (assume $\mathrm{K}$ is set to 1.5). Thus, upon hearing the route request from node $\mathrm{n}_{1}, \mathrm{n}_{10}$ issues a high-power route request which reaches node $n_{2}$. Node $n_{2}$ takes note that it receives a high-power route request from node $\mathrm{n}_{10}$ and rebroadcasts using regular power since the number of neighbors it observes exceeded $\mathrm{K}$. This goes on until the route request reaches node $\mathrm{D}$ which is the destination. When $\mathrm{n}_{2}$ receives a regular-power route reply from $\mathrm{n}_{5}$, it issues a high-power route reply to node $\mathrm{n}_{10}$ after attaching information regarding its $\left(\mathrm{n}_{2}\right)$ location and velocity. Node $n_{10}$ relays this route reply back to $S$ via node $\mathrm{n}_{1}$ using regular-power route reply after recording $\mathrm{n}_{2}$ 's location and velocity information. Since we assume that the data transmission rate is higher than the route request rate, we design the NDBAR scheme such that the node with low connectivity delivers the data packets via message ferrying. Thus, if this route is chosen, then when node $n_{10}$ receives data packets from node $n_{1}$, it will travel towards node $\mathrm{n}_{2}$ until it $\left(\mathrm{n}_{10}\right)$ is close enough to deliver the data packets via regular power transmission to node $\mathrm{n}_{2}$. Note that $\mathrm{n}_{10}$ can decide when it wants to move (e.g. after receiving several packets) towards $n_{2}$ depending on the speed of $n_{2}$ and the message rate that $\mathrm{n}_{10}$ receives from $\mathrm{n}_{1}$.

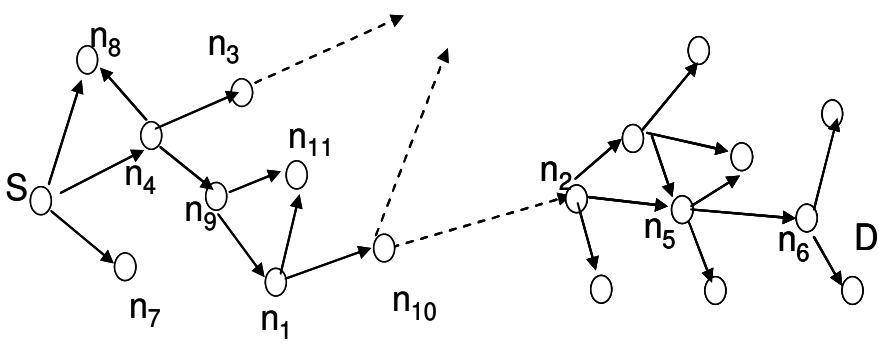

Figure 1. NDBAR scheme

\section{PERFORMANCE EVALUATION}

We implemented the NDBAR scheme using NS-2 network simulation package [8] and the simulation results are presented in this section. We also include the custody transfer feature described in [18],[25]. The transmission range is set at $250 \mathrm{~m}$ and each node is assumed to have 200 buffers. There are 10 flows and unless otherwise indicated, each flow has a packet rate of 1 every 4 seconds. The packet size is 512 bytes. The high power transmission is assumed to extend the transmission range to $500 \mathrm{~m}$. The performance metrics we use are:
- Packet delivery ratio (PDR) which is the number of packets that are correctly delivered to the destination over the number of unique data packets sent by the source.

- End-to-end delivery latency which is the time it takes to delivery a data packet. We consider both the average and the 95 percentile values.

- Hop count which is the average number of hops it takes for a data packet to arrive at the destination.

- Transmission overhead [19] which is defined as the number of transmitted bytes over the number of generated bytes. The transmission bytes include the routing overhead messages and custody transfer request and acknowledgment messages. Custody transfer and acknowledgment messages are assumed to be 35 bytes each.

We conducted several sets of experiments. For mobility, we either use the random waypoint model (RWP) [7] or the ZebraNet mobility model [19]. For the random waypoint model, each node moves towards a randomly picked destination at a constant speed. Once the destination is reached, another destination will be randomly chosen and the node will start moving towards the new destination after a certain pause time. This behavior is repeated for the whole duration of the simulation. In our simulation, the node's speed is chosen uniformly between zero and $5 \mathrm{~m} / \mathrm{s}$. For ZebraNet movement, we scale the node positions to fit into the geographical area used in our scenarios. We also scale the sampling time to be 8 seconds rather than 8 minutes. All the reported delay values in this paper are in seconds.

\section{A. Simulation Results}

\section{1) Impact of Node Density}

In our first set of experiments, we have 40 nodes distributed randomly over (a) $3000 \times 3000 \mathrm{~m}^{2}$, (b) $4000 \times 4000 \mathrm{~m}^{2}$, and (c) 5000x5000 $\mathrm{m}^{2}$. Table 2 tabulates the results. It shows that NDBAR can significantly improve the delivery ratio but it comes at the expense of transmission overhead. To improve the delivery ratio from $54.6 \%$ (refers to Table 1) to $96.2 \%$ (refers to Table 2) for the $3000 \times 3000 \mathrm{~m}^{2}$ scenario, one has to pay a transmission overhead of 17.5. It is almost impossible to deliver packets for case (c) using only multihop routing with custody transfer feature turned on but the NDBAR scheme can achieve a delivery ratio of $81.5 \%$ using a transmission overhead of 3.1 . 
Table 2: Performance of NDBAR scheme (RWP)

\begin{tabular}{|l|l|l|l|l|}
\hline Simulation Area & Delivery Ratio & Delay & Hop count & Overhead \\
\hline $3000 \times 3000$ & $96.2 \%$ & $818 \mathrm{sec}$ & 7.2 & 17.5 \\
\hline $4000 \times 4000$ & $95.5 \%$ & $1688 \mathrm{sec}$ & 5.3 & 12.4 \\
\hline $5000 \times 5000$ & $81.5 \%$ & $3455 \mathrm{sec}$ & 2.8 & 3.10 \\
\hline
\end{tabular}

To reduce the transmission overhead, we consider a variant of NDBAR (referred to as NDBARII). Each node is assumed to exchange information of its 1-hop and 2-hop neighbors with its immediate neighbors. A source node sends the data packet directly to a node that can reach the destination without going through the route request procedure. Otherwise, it broadcasts a data packet setting a neighbor relay expiry timer, w (set to 2000s). If a packet can be delivered to its destination via neighbor relaying before $w$ expires, then a route request will not be issued. During the neighbor relaying period, a node will send the data packet to a neighbor with the highest contact probability to the destination. Otherwise, a route request will be issued by the node which receives the data packet. If the message or route request is received by a node that does not have enough neighbors, then the node is allowed to issue a high power route request message. Each node maintains its contact probabilities with its 1 hop and 2 hop neighbors. Let us denote node $i$ 's contact probability with node $j$ as $P_{j}^{i}$. $P_{j}^{i}$ is updated as follows: node $i$ periodically broadcasts a neighbor discovery message; if node $i$ hears a response back from node $j$, then $P_{j}^{i}$ is set to 1 ; otherwise the existing $P_{j}^{i}$ value decays by a factor $\alpha$ (set to 0.8 in our experiments) periodically. We refer to this variant as the NDBAR-II scheme. We illustrate NDBAR-II in Figure 2. The source node $S$ attempts to deliver the packet to destination $\mathrm{D}$ initially via 2-hop relaying until the packet reaches node $n_{3}$ where the number of neighbors drops below the threshold so a high power route request is issued.

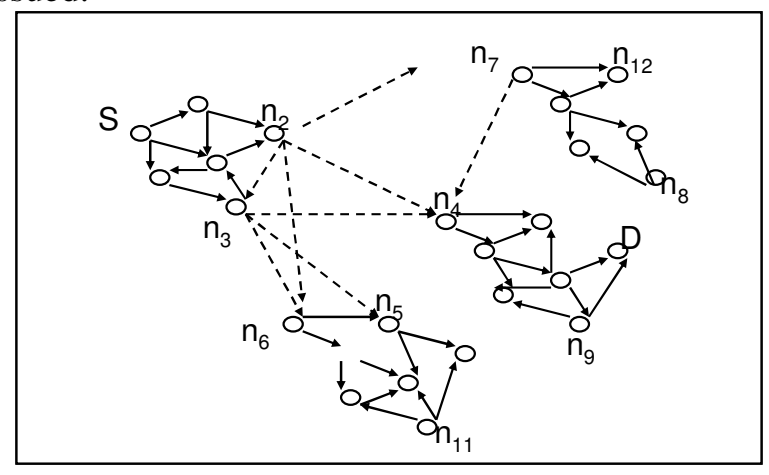

Figure 2: Enhanced NDBAR scheme (NDBAR-II)
The simulation results for the NDBAR-II scheme is shown in Table 3. Our results show that the NDBAR-II scheme can achieve relatively high delivery ratio (over $95 \%$ in $3000 \times 3000 \mathrm{~m}^{2}$ scenario) but with much reduced transmission overhead (decreases from 17.5 to 7.4 ). This comes at the cost of increasing the $95 \%$ message delivery latency. The transmission overhead will reduce further if higher packet generation rate is used.

\begin{tabular}{|l|l|l|l|l|l}
\hline $\begin{array}{l}\text { Simulation } \\
\text { Area }\end{array}$ & $\begin{array}{l}\text { Delivery } \\
\text { Ratio }\end{array}$ & Avg Dly & $\begin{array}{l}95 \% \\
\text { Pkt dly }\end{array}$ & Hop Count & Overhead \\
\hline $1000 \times 1000$ & $99.0 \%$ & 203 & 700 & 2.4 & 5.3 \\
\hline $2000 \times 2000$ & $96.4 \%$ & 1470 & 4000 & 2.3 & 4.3 \\
\hline $3000 \times 3000$ & $93.9 \%$ & 2465 & 4327 & 5.4 & 7.4 \\
\hline $4000 \times 4000$ & $92.4 \%$ & 2654 & 5123 & 4.3 & 7.2 \\
\hline
\end{tabular}

Table 3: Network performance (RWP) using NDBAR-II scheme.

2) Comparison with 2-hop erasure-coding and multihop schemes

In this section, we compare the routing performance of 2-hop erasure-coding relaying scheme [19], [25], the multihop routing scheme with custody transfer [18], and the NDBAR-II scheme. We simulated the scenarios tabulated in Table 4.

\begin{tabular}{|c|c|}
\hline Parameter & Value \\
\hline Simulation area & $\begin{array}{c}\text { 1000x1000, 1500x1500, 2000x2000, } \\
3000 \times 3000,4000 \times 4000\end{array}$ \\
\hline Simulation time & 5000 seconds \\
\hline Traffic pattern & 10 pairs of CBR 512 byte/pkt \\
\hline Mobility model & $\begin{array}{c}\text { RWP with maximum speed equal to } \\
5 \mathrm{~m} / \mathrm{s}, \text { Zebranet Mobility Pattern }\end{array}$ \\
\hline
\end{tabular}

Table 4: Simulation Parameters

Tables 5, 6 \& 7 tabulate the results for the 2-hop erasure-coding relay approach, the multihop approach and the NDBAR approach respectively with movements based on the random waypoint mobility model. Tables $8,9 \& 10$ tabulate the results for the three schemes using Zebranet mobility model [19].

Our results reveal that the 2-hop approach provides relatively good performance if ZebraNet mobility model is used until the node density drops below $4.4 \times 10^{-6}$ $\left(3000 \times 3000 \mathrm{~m}^{2}\right.$ scenario) when the delivery ratio becomes $76.2 \%$. However, the 2-hop approach achieves very poor performance when the random waypoint model is used as the mobility model achieving only $78.4 \%$ in the $1500 \times 1500 \mathrm{~m}^{2}$ scenario and only $41.9 \%$ in the $3000 \times 3000 \mathrm{~m}^{2}$ scenario. The delivery ratio with multihop approach is very good until the node density drops below $4.4 \times 10^{-6}$. The results clearly show that the NDBAR-II scheme achieves the best delivery ratio even 
in very spare networks and with reasonable transmission overhead. It also shows that NDBAR-II scheme is flexible enough to handle different mobility models.

\begin{tabular}{|c|c|c|c|c|c|}
\hline $\begin{array}{c}\text { Simulation } \\
\text { Area }\end{array}$ & $\begin{array}{c}\text { Delivery } \\
\text { Ratio }\end{array}$ & $\begin{array}{c}\text { Avg } \\
\text { Dly }\end{array}$ & $\begin{array}{c}95 \% \\
\text { Pkt } \\
\text { Dly }\end{array}$ & Overhead & $\begin{array}{c}\text { Hop } \\
\text { Count }\end{array}$ \\
\hline $1000 \times 1000$ & $95.3 \%$ & 381 & 950 & 9.84 & 2 \\
\hline $1500 \times 1500$ & $78.4 \%$ & 1724 & 2250 & 7.73 & 2 \\
\hline $2000 \times 2000$ & $41.9 \%$ & 1222 & 3300 & 6.23 & 2 \\
\hline $3000 \times 3000$ & $15.4 \%$ & 1224 & 4196 & 4.53 & 2 \\
\hline
\end{tabular}

Table 5: Delivery Performance using 2-hop approach (RWP)

\begin{tabular}{|c|c|c|c|c|c|}
\hline $\begin{array}{c}\text { Simulation } \\
\text { Area }\end{array}$ & $\begin{array}{c}\text { Delivery } \\
\text { Ratio }\end{array}$ & $\begin{array}{c}\text { Avg } \\
\text { Dly }\end{array}$ & $\begin{array}{c}95 \% \\
\text { Pkt Dly }\end{array}$ & Overhead & $\begin{array}{c}\text { Hop } \\
\text { Count }\end{array}$ \\
\hline $1000 \times 1000$ & $100 \%$ & 1.59 & 2.4 & 10.5 & 3.9 \\
\hline $1500 \times 1500$ & $99.99 \%$ & 43.4 & 2.7 & 13.2 & 5.7 \\
\hline $2000 \times 2000$ & $99.99 \%$ & 259.4 & 1600 & 10.4 & 5.3 \\
\hline $3000 \times 3000$ & $54.6 \%$ & 1829 & 3500 & 2.1 & 1.1 \\
\hline
\end{tabular}

Table 6: Delivery Performance using multihop approach (RWP)

\begin{tabular}{|c|c|c|c|c|c|}
\hline $\begin{array}{c}\text { Simulation } \\
\text { Area }\end{array}$ & $\begin{array}{c}\text { Delivery } \\
\text { Ratio }\end{array}$ & $\begin{array}{c}\text { Avg } \\
\text { Dly }\end{array}$ & $\begin{array}{c}95 \% \\
\text { Pkt dly }\end{array}$ & Overhead & $\begin{array}{c}\text { Hop } \\
\text { Count }\end{array}$ \\
\hline $1000 \times 1000$ & $99.0 \%$ & 203 & 700 & 5.3 & 5.32 .4 \\
\hline $2000 \times 2000$ & $96.4 \%$ & 1470 & 4000 & 4.3 & 4.32 .3 \\
\hline $3000 \times 3000$ & $93.9 \%$ & 2465 & 4327 & 7.4 & 7.45 .4 \\
\hline $4000 \times 4000$ & $92.4 \%$ & 2654 & 5123 & 7.2 & 7.24 .3 \\
\hline
\end{tabular}

Table 7: Delivery Performance using NDBAR-II (RWP)

\begin{tabular}{|c|c|c|c|c|c|}
\hline $\begin{array}{c}\text { Simulation } \\
\text { Area }\end{array}$ & $\begin{array}{c}\text { Delivery } \\
\text { Ratio }\end{array}$ & $\begin{array}{c}\text { Avg } \\
\text { Dly }\end{array}$ & $\begin{array}{c}95 \% \\
\text { Pkt Dly }\end{array}$ & Overhead & $\begin{array}{c}\text { Hop } \\
\text { Count }\end{array}$ \\
\hline $1000 \times 1000$ & $96.8 \%$ & 710 & 420 & 7.0 & 2.0 \\
\hline $1500 \times 1500$ & $96.5 \%$ & 366 & 950 & 8.0 & 2.0 \\
\hline $2000 \times 2000$ & $99.0 \%$ & 128 & 1500 & 10.3 & 2.0 \\
\hline $3000 \times 3000$ & $76.2 \%$ & 1678 & 4312 & 5.1 & 2.0 \\
\hline
\end{tabular}

Table 8: Delivery Performance using 2-hop approach (ZebraNet)

\begin{tabular}{|c|c|c|c|c|c|}
\hline $\begin{array}{c}\text { Simulation } \\
\text { Area }\end{array}$ & $\begin{array}{c}\text { Delivery } \\
\text { Ratio }\end{array}$ & $\begin{array}{c}\text { Avg } \\
\text { Dly }\end{array}$ & $\begin{array}{c}95 \% \\
\text { Pkt } \\
\text { Dly }\end{array}$ & Overhead & $\begin{array}{c}\text { Hop } \\
\text { Count }\end{array}$ \\
\hline $1000 \times 1000$ & $99.99 \%$ & 0.71 & 3 & 9.3 & 2.1 \\
\hline $1500 \times 1500$ & $99.99 \%$ & 29 & 130 & 14.4 & 4.1 \\
\hline $2000 \times 2000$ & $99.99 \%$ & 203 & 700 & 14.8 & 3.5 \\
\hline $3000 \times 3000$ & $89.4 \%$ & 1146 & 3985 & 2.47 & 1.1 \\
\hline
\end{tabular}

Table 9: Delivery Performance using multihop approach (ZebraNet)

\begin{tabular}{|c|c|c|c|c|c|}
\hline $\begin{array}{c}\text { Simulation } \\
\text { Area }\end{array}$ & $\begin{array}{c}\text { Delivery } \\
\text { Ratio }\end{array}$ & $\begin{array}{c}\text { Avg } \\
\text { Delay }\end{array}$ & $\begin{array}{l}95 \% \\
\text { Pkt } \\
\text { Delay }\end{array}$ & Overhead & $\begin{array}{c}\text { Hop } \\
\text { Count }\end{array}$ \\
\hline $1000 \times 1000$ & $98.7 \%$ & 86.9 & 488 & 5.96 & 2.9 \\
\hline $2000 \times 2000$ & $98.0 \%$ & 746 & 3000 & 4.64 & 2.6 \\
\hline $3000 \times 3000$ & $93.2 \%$ & 1242 & 3899 & 8.6 & 2.4 \\
\hline $4000 \times 4000$ & $90.4 \%$ & 2018 & 4565 & 7.7 & 2.8 \\
\hline
\end{tabular}

Table 10: Delivery Performance using NDBAR-II (ZebraNet)

\section{3) Impact of Traffic Model}

We next evaluate the impact of traffic model on delivery performance using NDBAR-II scheme and random waypoint mobility model. In earlier sections, we use CBR traffic model for all the flows. In this section, we use a bidirectional traffic model described in [16],[17]. The source sends a message to the destination. Upon receiving the message, the destination will respond with another message. Random waypoint mobility model is used in this set of experiments. We evaluate the endto-end delay of the bidirectional message flows.

Table 11 tabulates our simulation results. Compared to Table 7, the $95 \%$ bidirectional message delay is only about $10 \%$ higher than the $95 \%$ unidirectional message delay when the node density is above $4.4 \times 10^{-6}$ but it almost doubles when the node density decreases to $4.4 \times 10^{-6}$ and it triples when the node density drops to $2.5 \times 10^{-6}$. The delivery ratio has dropped to $85.3 \%$ with bidirectional flows when the node density is $2.5 \times 10^{-6}$ but it is still relatively high. The transmission overhead improves since the messages in the reverse direction do not have to incur extra route discovery overhead.

\begin{tabular}{|c|c|c|c|c|c|}
\hline $\begin{array}{c}\text { Simulation } \\
\text { Area }\end{array}$ & $\begin{array}{c}\text { Bidirectional- } \\
\text { Delivery } \\
\text { Ratio }\end{array}$ & $\begin{array}{c}\text { Avg } \\
\text { Delay }\end{array}$ & $\begin{array}{l}95 \% \\
\text { Pkt } \\
\text { Delay }\end{array}$ & Overhead & $\begin{array}{c}\text { Hop } \\
\text { Count }\end{array}$ \\
\hline $1000 \times 1000$ & $99.0 \%$ & 260 & 734 & 3.3 & 4.8 \\
\hline $2000 \times 2000$ & $96.4 \%$ & 533 & 4417 & 2.7 & 4.3 \\
\hline $3000 \times 3000$ & $91.0 \%$ & 4576 & 8643 & 9.1 & 9.6 \\
\hline $4000 \times 4000$ & $85.3 \%$ & 6414 & 15435 & 6.6 & 7.4 \\
\hline
\end{tabular}

Table 11: Delivery Performance using NDBAR-II (RWP)

\section{CONCLUSION}

In this paper, we have designed a new routing scheme called the Node Density Based Adaptive Routing (NDBAR) scheme for DTN environment. Our scheme makes use of the neighbor density information each node observes to decide when a node will function as a message ferry to deliver data packets. Our preliminary simulation results indicate that both the 2hop erasure-coding relay approach and the multihop with custody transfer approach fail to provide reasonable delivery ratio when the node density is lower than $4.4 \times 10^{-6}$ (equivalent to 40 nodes over $3000 \times 3000 \mathrm{~m}^{2}$ with a regular transmission range of $250 \mathrm{~m}$ ). The multihop with custody transfer approach provides better delivery ratio than the 2-hop erasure-coding approach but incurs higher transmission overhead. The NDBAR scheme that we design provides the best delivery ratio 
but this comes at the cost of additional transmission overhead. The enhanced NDBAR scheme (NDBAR-II) scheme that we designed is able to achieve comparable delivery ratio performance as the NDBAR scheme but with much reduced transmission overhead. Our simulation results indicate that the NDBAR-II scheme can achieve more than $90 \%$ delivery ratio using ZebraNet mobility model and $92 \%$ delivery ratio with a node density of $2.5 \times 10^{-6}$ when the transmission range is $250 \mathrm{~m}$. With bidirectional flows, our NDBAR-II scheme can still achieve $85.3 \%$ under the same network conditions. We intend to implement the NDBAR-II scheme and evaluate its performance in a reasonable size testbed.

\section{ACKNOWLEDGMENT}

. This work is sponsored by Defense Advanced Research Projects Agency (DARPA) under contract W15P7T-05-CP413. Any opinions, findings, and conclusions or recommendations expressed in this material are those of the authors and do not necessarily reflect the views of DARPA. This document is approved for public release, unlimited distribution.

\section{REFERENCES}

[1] Y. Gitman, "Magic Bike: Wireless Internet", http://www.magicbike.net/

[2] L. Arent, G4techTV, Tech-Live, "Wi-Fi on Two Wheels", http://www.g4techtv.com/techtvvault/features/47657/WiF i_on_Two_Wheels.html, May 2004

[3] A. Pentland, R. Fletcher, and A. Hasson, "DakNet: Rethinking connectivity in developing nations", IEEE Computer, 37(1):78-83, January 2004.

[4] H. Balakrishnan, V. N. Padmanabhan, S. Seshan, and R. H. Katz, "A comparison of mechanisms for improving TCP performance over wireless links", IEEE/ACM Transactions on Networking, 5(6):756-769, December 1997.

[5] K. Fall, "A delay-tolerant network architecture for challenged Internets", Proceedings of SIGCOMM'03, August 2003.

[6] M. Chuah, L. Cheng, and B. D. Davison, "Enhanced Disruption and Fault Tolerant Network Architecture for Bundle Delivery (EDIFY)", to appear in Globecom, 2005

[7] J. Broch etc, "A performance comparison of multihop wireless adhoc network routing protocol", Proceedings of Mobicom, 1998.

[8] UCB/LBNL/VINT, "The Network Simulator ns-2", Online at http://www.isi.edu/nsnam/ns/

[9] J. H. Saltzer, D. P. Reed, and D. D. Clark, "End-to-End Arguments in System Design", ACM Transactions on Computer Systems, 2(4):277-288, November 1984

[10] J. Klensin, Editor, "Simple mail transfer protocol", IETF RFC 2821, April 2001.
[11] R. Viswanathan, J. Li, and M. C. Chuah, "Message Ferrying for Constrainted Scenarios", poster, Proceedings of WoWMoM, 2005.

[12] W. Zhao and M. H. Ammar, "Message Ferrying: Proactive Routing in Highly Partitioned Wireless Adhoc Networks", Proceedings of the IEEE Workshop on Future Trends in Distributed Computing Systems, Puerto Rico, May, 2003.

[13] V. Cerf et al, "Delay-Tolerant Network Architecture", Internet Draft, draft-irtf-dtnrg-arch-02.txt, July 2004.

[14] B. Burns, O. Brock and B. N. Levine, "MV Routing and Capacity Building in Disruption Tolerant Networks", IEEE Infocom, March 2005.

[15] W. Zhao, M. Ammar, and E. Zegura, “A message ferrying approach for data delivery in sparse mobile ad hoc networks", ACM Mobihoc, May 2004.

[16] W. W. Brown, T. Krout, "Future Performance Expectations for Mobile Wireless Communication Networks", preprint, 2005

[17] J. Hsu, S. Bhatia, M. takai, R. Bagrodia, M. J. Acriche, "Performance of Mobile Adhoc Networking Routing Protocols in Realistic Scenarios", Milcom 2003.

[18] K. Fall, W. Hong and S. Madden, "Custody transfer for reliable delivery in delay tolerant networks", IRB-TR-03030, July 2003.

[19] Y. Wang etc, "Erasure-Coding Based Routing for Opportunistic Networks", Proceedings of Sigcom WDTN workshop, 2005.

[20] R. Shah etc, "Data mules: Modeling and analysis of a three-tier architecture for sparse sensor networks", Elsevier Ad Hoc Networks Journal, Vol 1, pp 215-233, Sept, 2003

[21] P. Juang, etc, "Energy-efficient computing for wildlife tracking: design tradeoffs and early experiences with zebranet", Proc ASPLOS, Oct 2002.

[22] A. Lindgren, A. Doria, O. Schelen, "Probabilistic routing in intermittently connected networks", Sigmobile, Mobile Computing and Communications Review, Vol 7(3), pp 19-20, 2003.

[23] S. Jain, M. Demmer, R. Patra, K. Fall, "Using Redundancy to cope with Failures in a Delay Tolerant Network", Proceedings of Sigcomm 2005.

[24] A. Vahdat, D. Becker, "Epidemic Routing for partially connected adhoc networks", Tehnical Report CS-200006, Duke University, April 2000.

[25] M.Chuah, P. Yang, B. Davison, L. Cheng, "Performance Evaluation of a Multihop Unicast Delivery Scheme for DTNs", Darpa DTN Technical Report for EDIFY project, Lehigh Univeristy, Oct, 2005.

[26] J. Burgess, B. Gallagher, D. Jensen, B.N. Levine, "MaxProp: Routing for Vehicle-Based DisruptionTolerant Networking", to appear in Proceedings of IEEE Infocom,April,2006. 